\title{
A Facile Method for Preparing Transparent, Conductive, and Paper-Like Silver Nanowire Films
}

\author{
Yajie Wang, Tao Feng, Kai Wang, Min Qian, Yiwei Chen, and Zhuo Sun \\ Department of Physics, Engineering Research Center for Nanophotonics and Advanced Instrument, Ministry of Education, \\ East China Normal University, Shanghai 200062, China \\ Correspondence should be addressed to Tao Feng, tfeng@phy.ecnu.edu.cn
}

Received 18 April 2011; Accepted 1 July 2011

Academic Editor: Yanqiu Zhu

Copyright (C) 2011 Yajie Wang et al. This is an open access article distributed under the Creative Commons Attribution License, which permits unrestricted use, distribution, and reproduction in any medium, provided the original work is properly cited.

Transparent, conductive, and flexible silver nanowire (AgNW) films have been fabricated by a facile two-step method. Firstly, the well-dispersed AgNW suspension is vacuum filtered using mixed esters of cellulose (MCE) membranes as filters. Then, the AgNW-MCE films are treated with acetone vapor. After the infiltration of acetone vapor, the white and porous MCE membranes change into transparent and pore-free, and AgNW-MCE films are obtained with extraordinary optical, conductive, and mechanical properties. An optimal result is obtained with transmittance of $85 \%$ at $550 \mathrm{~nm}$ and sheet resistance about $50 \mathrm{Ohm} / \mathrm{sq}$. The flexibility of AgNW-MCE films is remarkable, which is comparable to that of the AgNW film on flexible polyethylene terephthalate (PET). More important, AgNW-MCE films show an excellent adhesion to the substrate, which causes a stable electrical conductivity even after scotch tape test and finger friction test. As a result of improved adhesion to the substrate, the sheet resistance of AgNW-MCE films is about $20 \%$ smaller than that of AgNW-PET films.

\section{Introduction}

Nowadays, as optoelectronic technology will move toward flexible and paper-like devices, great efforts have been made to search for suitable electrode materials which can be formed into films at low temperature and used in such applications. As we all know, indium tin oxide (ITO) as the most important transparent and conductive film (TCF) has been widely used in optoelectronics for 50 years, but it is completely unsuitable to flexible devices because of its brittle nature [1]. To our excitement, many promising materials have been emerging such as graphenes, carbon nanotubes, and metal nanowires [2-6]. For these materials, one of the most important advantages is that the flexibility does not come at the cost of performance in terms of sheet resistance and transparency [7]. Among these materials, random networks of AgNW, owing to its highest electrical conductivity and optical transparency, are the most promising replacement for ITO [4-6]. More recently, flexible AgNW electrodes have been fabricated and successfully used in organic solar cells [8] and organic light emitting devices [9], and the performance of the electrode in terms of sheet resistance and transparency can match ITO. However, to be used in optoelectronic devices, the AgNW film should have a strong adhesion to the substrate, which is hard to achieve while AgNW films deposited on plastic substrate without further treatments [4]. Although some methods including encapsulation [5] and burying AgNW networks [9] have been taken to resolve the adhesion issue, both methods need more complicated steps after AgNW films prepared. So, a simple method to prepare flexible AgNW films with good adhesion is still urgently required.

Vacuum filtration is a low-temperature process in film production, which affords films with some advantages such as surface uniformity, high electric conductivity, and controllable thickness. Therefore, it has been widely used for fabricating AgNW films $[4,5]$. To get transparent electrodes, AgNW films need to be transferred to a transparent substrate after deposited on the filter, but this process is usually complicated. In this paper, we use the hot block technique [10] to prepare transparent, conductive, and paper-like AgNW-MCE films with outstanding adhesion. As the mixed 


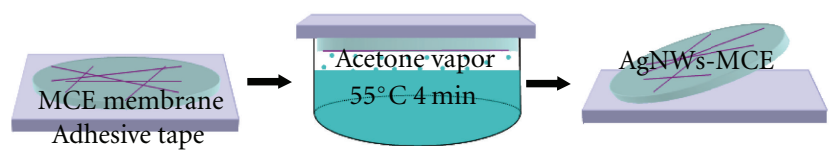

FIGURE 1: Schematic illustration of the preparing AgNW-MCE films process.

esters of cellulose (MCE) membrane, used as the filter, can become transparent and pore-free [11], the transfer process would be eliminated.

\section{Experimental}

Silver nanowires (average diameter of $90 \mathrm{~nm}$, average length of $10 \mu \mathrm{m}$ ) were purchased from Blue Nano as suspensions with a concentration of $10 \mathrm{mg} / \mathrm{mL}$ in ethanol. The dispersion was further diluted down to $0.027 \mathrm{mg} / \mathrm{mL}$ with deionized water and then sonicated for $20 \mathrm{~min}$. AgNW films were prepared by vacuum filtration with various volumes of nanowire solution using porous MCE membranes $(0.45 \mu \mathrm{m}, 47 \mathrm{~mm})$ and then dried at room temperature.

2.1. Preparation of AgNW-MCE Films. The deposited film with AgNWs face up was placed on adhesive tapes and treated with acetone vapor for $4 \mathrm{~min}$. Then, the MCE membrane became transparent and pore-free. MCE membrane was peeled off from the tape after it was dried at room temperature. The entire process is very simple and just takes only a few minutes. Adhesive tape was used necessarily to prevent potential curling and minimize the shrinkage of MCE membrane [10]. Schematic representation of preparing AgNW-MCE films is illustrated in Figure 1.

2.2. Preparation of $A g N W$-PET Films. The deposited film was placed on the PET with the AgNWs in contact with the PET and then placed on a flat plate while heated at $100^{\circ} \mathrm{C}$ on the bottom and pressed with a pressure of $0.6 \mathrm{MPa}$ on the top for $20 \mathrm{~min}$. MCE membrane was removed by treatment with acetone vapor for $4 \mathrm{~min}$ and subsequent acetone liquid baths for $20 \mathrm{~min}$, and then, AgNW films were transferred to PET [12].

2.3. Characterization of Samples. The morphology of AgNWMCE films and AgNW-PET films was characterized by SEM using S-4700. Sheet resistance (Rs) of these films was tested by an RS-8 4-point probes resistivity measurement system. The optical transmittance of these films was measured by a U-3900 UV-VIS spectrophotometer, with a transparent MCE membrane as reference.

For adhesion and flexibility comparison, the AgNWMCE film and the AgNW-PET film prepared with the same volume $(6 \mathrm{~mL})$ were pressed under a pressure of $0.6 \mathrm{MPa}$ for $20 \mathrm{~min}$. Then, the adhesion test of those films was performed using a Scotch tape [6]. Tapes were put gently on the sample surface and pressed under a pressure of $0.6 \mathrm{MPa}$ for $10 \mathrm{sec}$ then were peeled off.

\section{Results and Discussion}

As seen in the photographs shown in Figure 2, the color of the MCE membrane is changed from white (Figure 2(a)) to transparent (Figure 2(b)). After treated with acetone vapor, the transmittance of the MCE membrane is over $90 \%$, which is high enough as a matrix of TCF. When infiltrated with acetone vapor, the MCE membrane became viscous, so its pores were melted [11]. As is shown in Figures 3(a) and $3(\mathrm{~b})$, there is no pore in the SEM image of AgNW-MCE films, and the morphology is similar to that of AgNWPET films (Figure 3(c)). It is clear from Figure 3(d) that the MCE membrane is porous before treated with acetone vapor. The SEM image in Figure 3(a) shows networks of nanowires with big holes that are above percolation. The percolation problem, which could be detrimental to some electrode applications, can be overcome effectively by incorporating other materials into AgNW networks, such as CNTs, PEDOT, and graphenes.

We prepared samples with various densities of silver nanowires, using $1.5 \mathrm{~mL}, 2 \mathrm{~mL}, 2.5 \mathrm{~mL}, 3 \mathrm{~mL}, 3.5 \mathrm{~mL}, 4 \mathrm{~mL}$, $5 \mathrm{~mL}$, and $6 \mathrm{~mL}$ volume of previous nanowire solution separately. It is evident from Figure 4(a) that optical transmittance decreases with increasing of AgNWs density. The sample using $1.5 \mathrm{~mL}$ of starting solution has the highest transmittance about $88 \%$. On the other hand, the Rs increases as the density increases, as shown in Figure 4(b). AgNW-MCE films with Rs around $50 \mathrm{Ohm} / \mathrm{sq}$ and $\sim 85 \%$ optical transmittance (at $550 \mathrm{~nm}$ ) were produced, which is very close to the performance of the ITO on PET for flexible optoelectronic devices. The properties of AgNW-MCE films show that they are suitable for the use of touch screen panels, electrowetting display, and liquid crystal display $[13,14]$. We have to note that all the Rs values of AgNW-MCE films, shown in Figure 4(b), were obtained after the film pressed under the pressure of $10 \mathrm{MPa}$, resulting in the Rs of the film significantly decreasing by about $50 \%$. We attributed the decrease to cutting down the contact resistance between AgNWs. The mechanical pressing method, reported by Hu et al. [6], is an available approach to reduce the junction resistance of AgNWs. They have reduced the Rs of the AgNW film from several hundreds ohms/sq to several tens ohms/sq after pressed up to $81 \mathrm{GPa}$ pressure. According to that result, we believe that the conductivity of AgNW-MCE films can be considerably improved by pressing films under a higher pressure.

For AgNW-MCE films, the mechanical robustness against adhesion, friction, and bending is excellent. The equivalence thickness of the AgNW film used for the adhesion and flexibility test is $300 \mathrm{~nm}$ (for the deposition density is $130 \mathrm{mg} / \mathrm{m}^{2}$ ) [4]. It is notable in Figure 4(c) that there is no obvious increase in Rs of AgNW-MCE films after tape test and finger friction. In contrast, the conductivity of AgNW-PET films was nearly lost while it was peeled off from the tape and rubbed against by finger. From the inset figure of Figure 4(c), it was noticeable that the AgNWs on PET were mostly peeled off (left 2) or rubbed away (left 3), while AgNW-MCE films were without any visible change. The reason for the outstanding adhesion is as follows. (1) While treated with acetone vapor, the MCE membrane became sticky. As a result, 


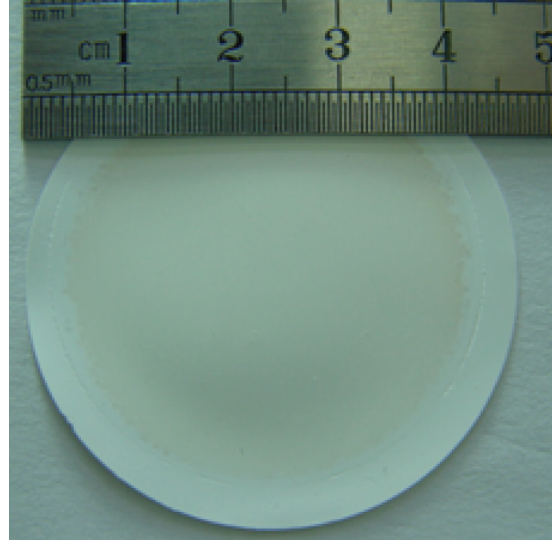

(a)

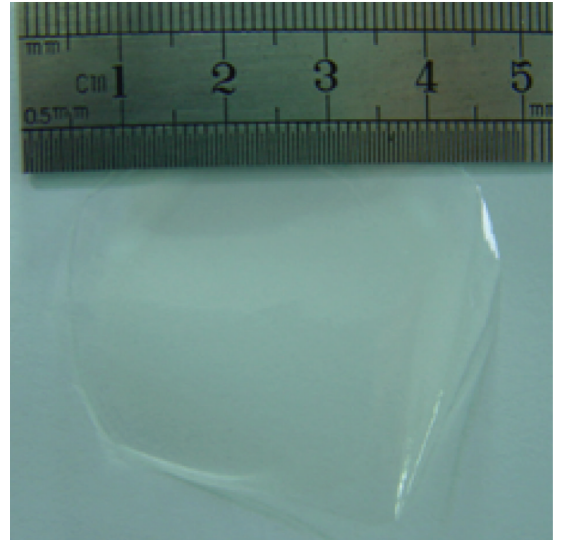

(b)

Figure 2: Photographs of a AgNW-MCE film (a) before and (b) after treated with acetone vapor.

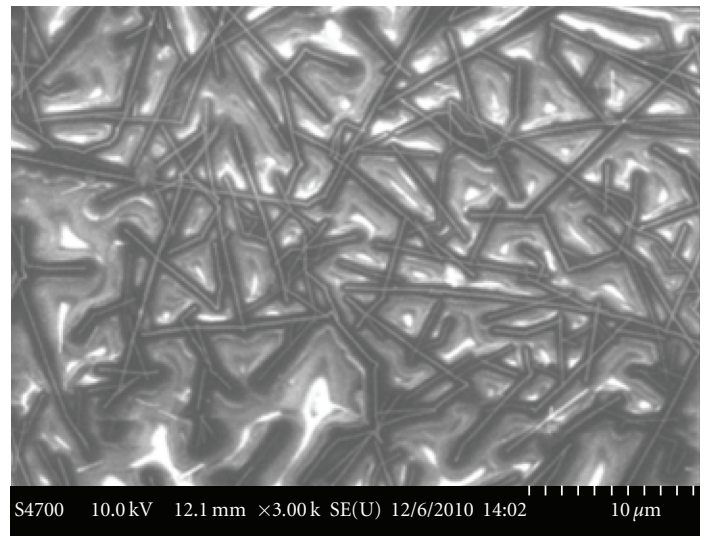

(a)

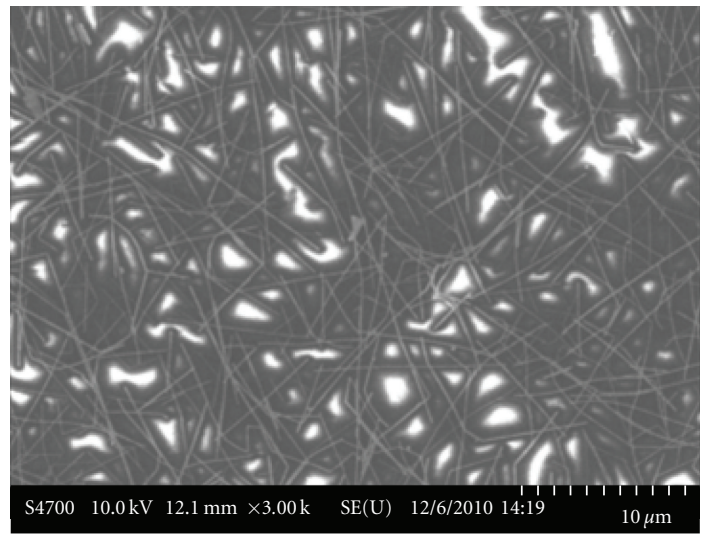

(c)

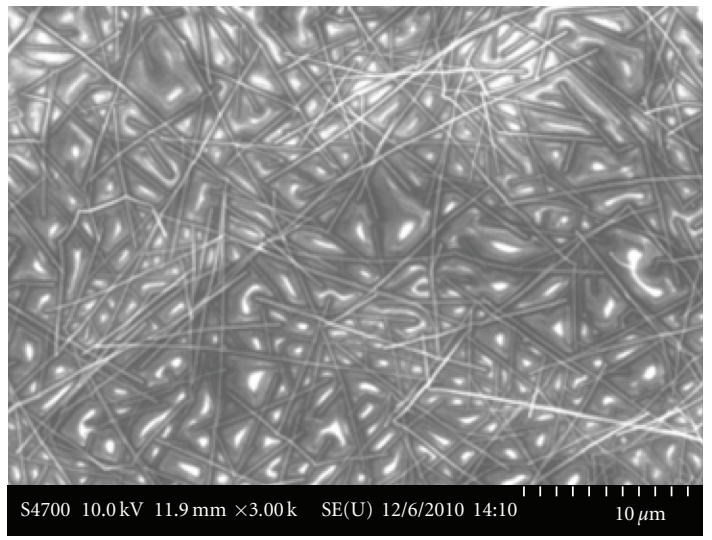

(b)

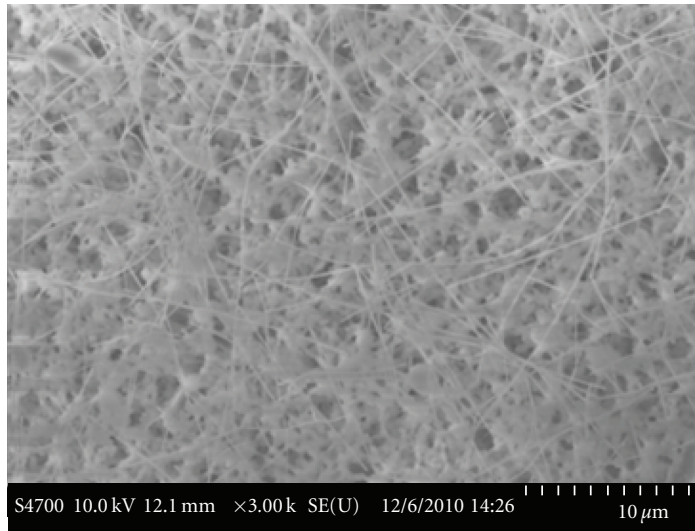

(d)

FIGURE 3: SEM images of AgNW-MCE films after treated with acetone vapor prepared using (a) $2 \mathrm{~mL}$ and (b) $6 \mathrm{~mL}$ volume of nanowire solution. (c) AgNW-PET films using $6 \mathrm{~mL}$ volume of nanowire solution. (d) AgNW-MCE films (6 mL) before treated with acetone vapor.

AgNWs were stuck to the MCE membrane. (2) The pore were melted after treated with acetone vapor; meanwhile, some ends of AgNWs were buried in the MCE membrane [10]. During the flexibility testing, as shown in Figure 4(d), the conductivity is nearly invariant while bending with the AgNW-MCE film in compress and tension. The films were bent to a curvature of $1-2 \mathrm{~mm}$ (the inset figure of Figure $4(\mathrm{~d})$ ). If noting the difference of Rs between AgNWMCE films and AgNW-PET films, as shown in Figure 4(d), we can see the Rs of AgNW-MCE films is about 20\% lower 


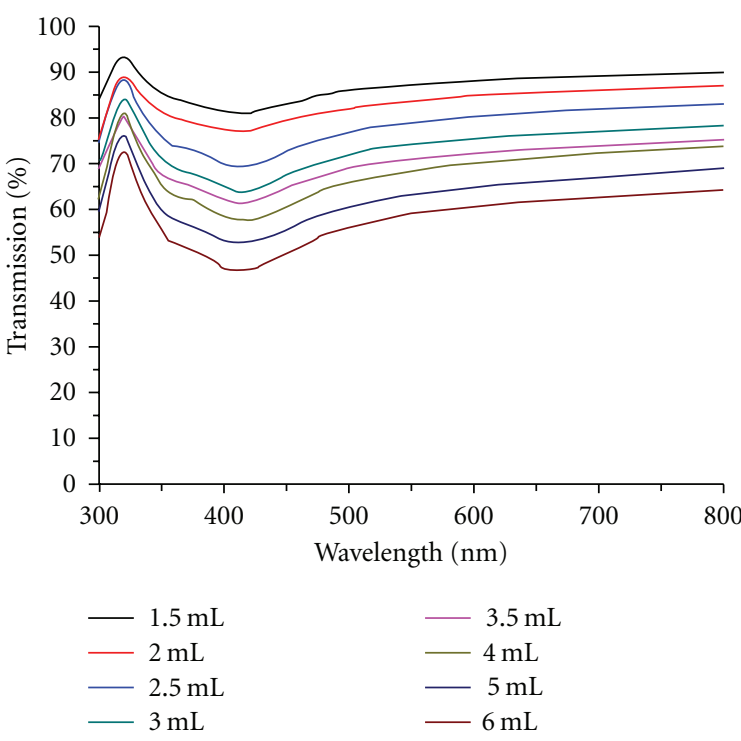

(a)

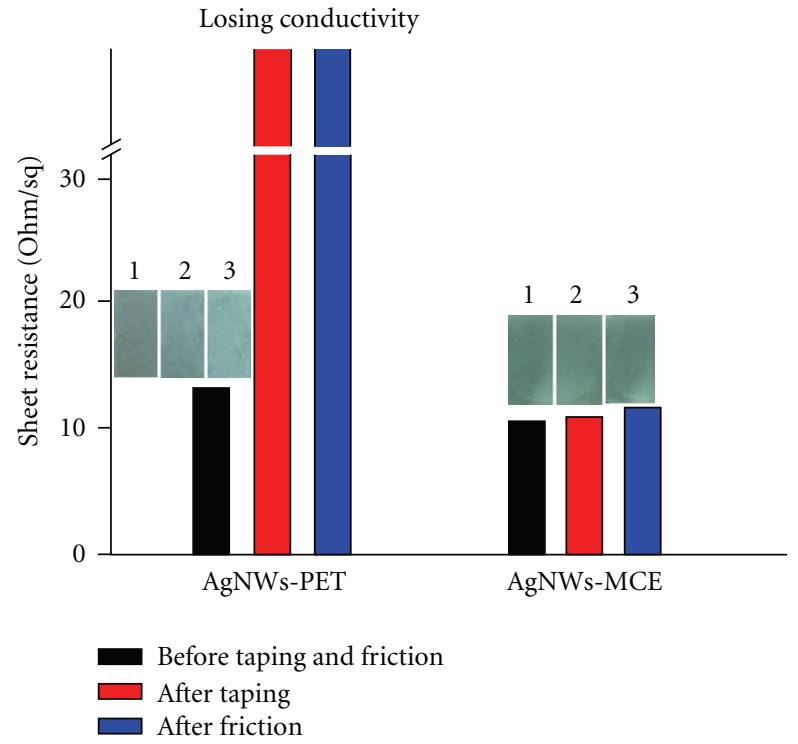

(c)

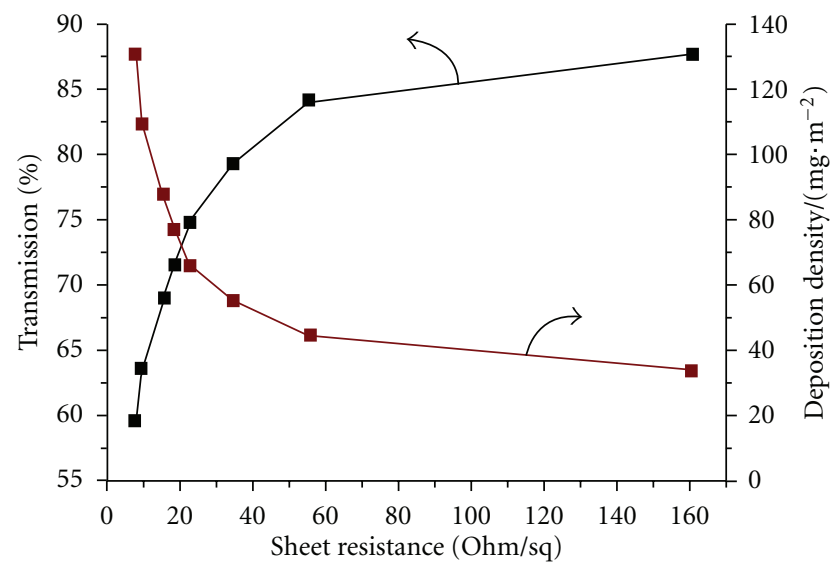

(b)

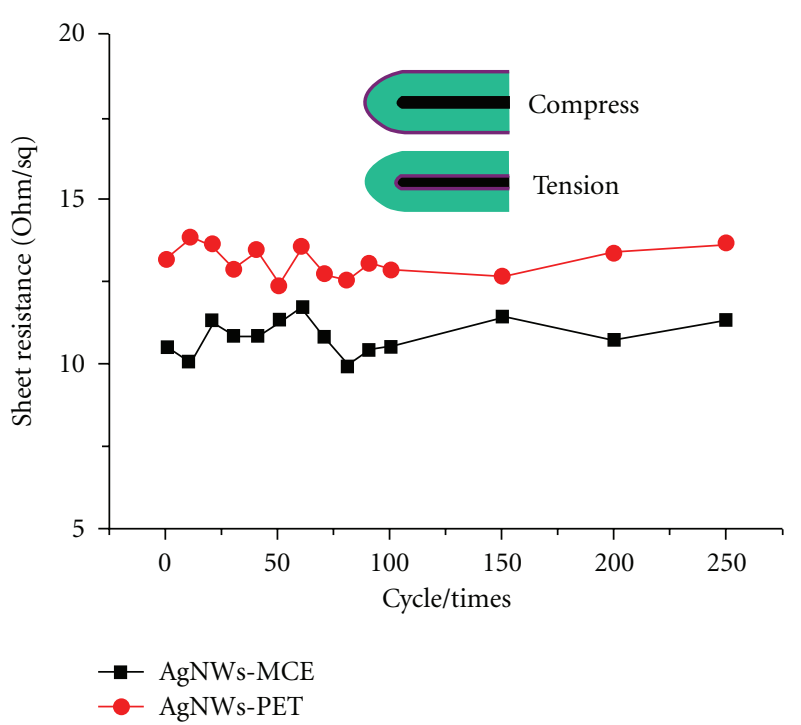

(d)

Figure 4: (a) Optical transmittance of AgNW-MCE films. (b) Rs versus transmission (at $550 \mathrm{~nm}$ ) and deposition density of AgNWs-MCE films. (c) The Rs of AgNW-MCE and AgNW-PET films before and after taping and friction test, respectively. The inset photos are AgNWPET films (left) and AgNW-MCE films (right) before (1) and after destroyed by tape adhesion (2) and finger friction (3). (d) The change in resistance of AgNW-MCE and AgNW-PET films after cycles of folding up.

than that of AgNW-PET films. That is possible because the contact between AgNWs to the substrate improved, which improves the morphology and the electrical contacts between wires [6].

\section{Conclusions}

In summary, we have demonstrated a simple, rapid, and lowcost method to fabricate transparent AgNW films. Unlike the AgNW film on PET with poor adhesion, the AgNW film prepared by this method adheres strongly on the MCE substrate, which causes a stable conductivity even if the film is subjected to bending. We have obtained AgNW-MCE films with Rs around $50 \mathrm{Ohm} / \mathrm{sq}$ and $\sim 85 \%$ visible transmittance, which is comparable to ITO on PET. As mentioned above, we believe that AgNW-MCE films can be widely used in flexible electronic and optoelectronic devices.

\section{Acknowledgments}

This work was supported by the Rising Star Program of Shanghai under Grant no. 07QA14019, Shanghai Talent Development Fund, and the Fundamental Research Funds for the Central Universities. 


\section{References}

[1] Z. Chen, B. Cotterell, and W. Wang, "The fracture of brittle thin films on compliant substrates in flexible displays," Engineering Fracture Mechanics, vol. 69, no. 5, pp. 597-603, 2002.

[2] X. Wang, L. Zhi, and K. Müllen, "Transparent, conductive graphene electrodes for dye-sensitized solar cells," Nano Letters, vol. 8, no. 1, pp. 323-327, 2008.

[3] L. B. Hu, W. Yuan, P. Brochu, G. Gruner, and Q. Pei, "Highly stretchable, conductive, and transparent nanotube thin films," Applied Physics Letters, vol. 94, no. 16, Article ID 161108, 3 pages, 2009.

[4] S. De, T. M. Higgins, P. E. Lyons et al., "Silver nanowire networks as flexible, transparent, conducting films: extremely high DC to optical conductivity ratios," ACS Nano, vol. 3, no. 7, pp. 1767-1774, 2009.

[5] A. R. Madaria, A. Kumar, F. N. Ishikawa, and C. Zhou, "Uniform, highly conductive, and patterned transparent films of a percolating silver nanowire network on rigid and flexible substrates using a dry transfer technique," Nano Research, vol. 3, no. 8, pp. 564-573, 2010.

[6] L. B. Hu, H. S. Kim, J. Y. Lee, P. Peumans, and Y. Cui, "Scalable coating and properties of transparent, flexible, silver nanowire electrodes," ACS Nano, vol. 4, no. 5, pp. 2955-2963, 2010.

[7] P. J. King, U. Khan, M. Lotya, S. De, and J. N. Coleman, "Improvement of transparent conducting nanotube films by addition of small quantities of graphene," ACS Nano, vol. 4, no. 7, pp. 4238-4246, 2010.

[8] J. Y. Lee, S. T. Connor, Y. Cui, and P. Peumans, "Semitransparent organic photovoltaic cells with laminated top electrode," Nano Letters, vol. 10, no. 4, pp. 1276-1279, 2010.

[9] X. Y. Zeng, Q. K. Zhang, R. M. Yu, and C. Z. Lu, "A new transparent conductor: silver nanowire film buried at the surface of a transparent polymer," Advanced Materials, vol. 22, no. 40, pp. 4484-4488, 2010.

[10] J. S. Webber, A. G. Czuhanich, and L. J. Carhart, "Performance of membrane filters used for TEM analysis of asbestos," Journal of Occupational and Environmental Hygiene, vol. 4, no. 10, pp. 780-789, 2007.

[11] G. J. Burdett and A. P. Rood, "Membrane-filter, direct-transfer technique for the analysis of asbestos fibers or other inorganic particles by transmission electron microscopy," Environmental Science and Technology, vol. 17, no. 11, pp. 643-648, 1983.

[12] Z. C. Wu, Z. H. Chen, X. Du et al., "Transparent, conductive carbon nanotube films," Science, vol. 305, no. 5688, pp. 12731276, 2004.

[13] L. B. Hu, G. Gruner, J. Gong, C. J. Kim, and B. Hornbostel, "Electrowetting devices with transparent single-walled carbon nanotube electrodes," Applied Physics Letters, vol. 90, no. 9, Article ID 093124, 3 pages, 2007.

[14] D. Hecht, L. B. Hu, and G. Grüner, "Conductivity scaling with bundle length and diameter in single walled carbon nanotube networks," Applied Physics Letters, vol. 89, no. 13, 3 pages, 2006. 

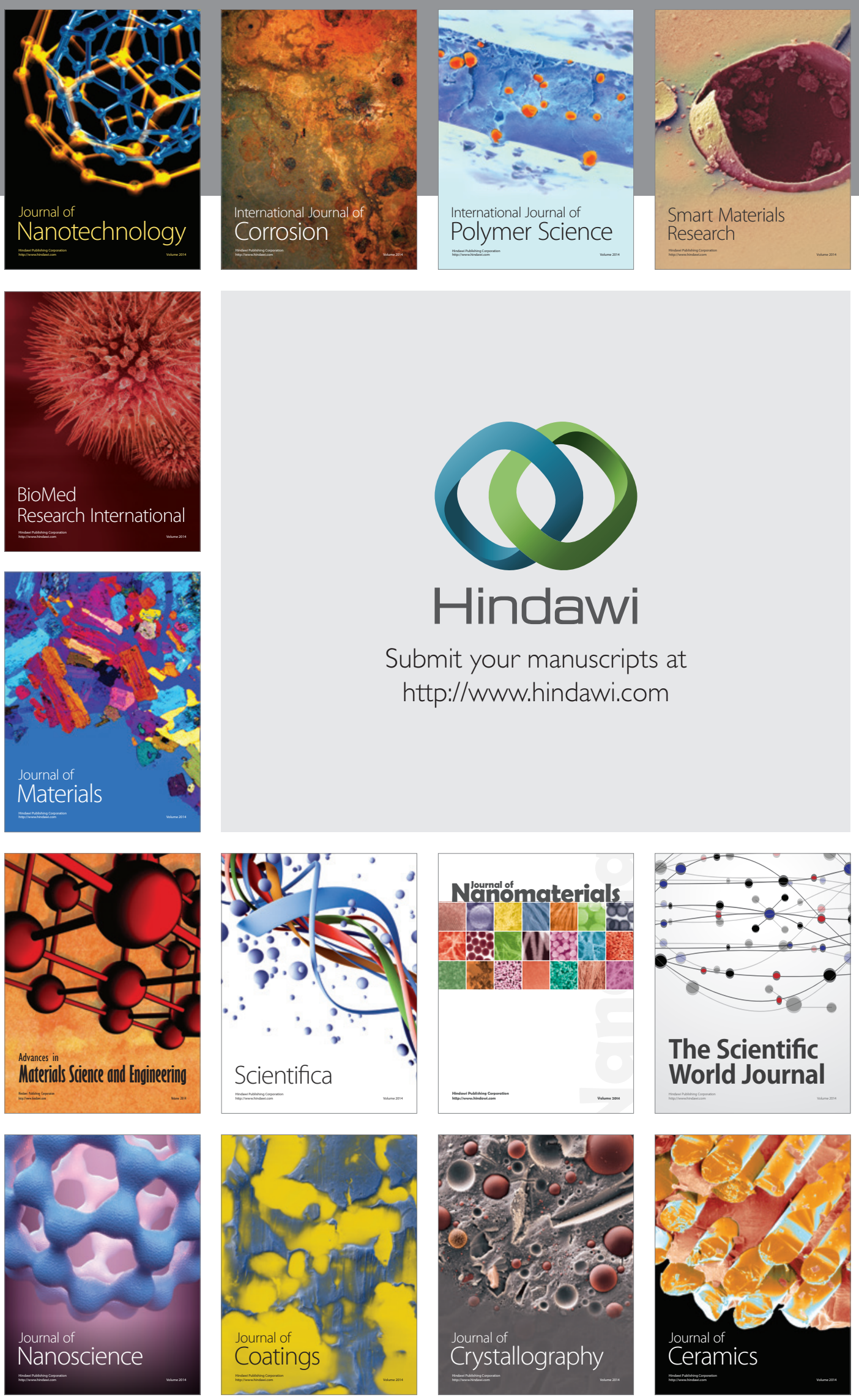

The Scientific World Journal

Submit your manuscripts at

http://www.hindawi.com

\section{World Journal}

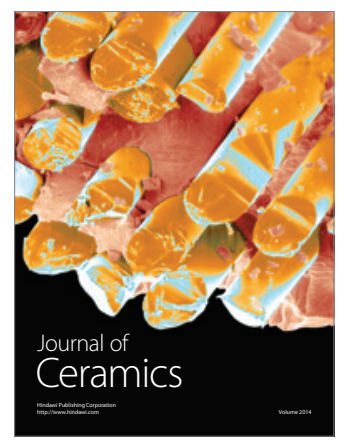

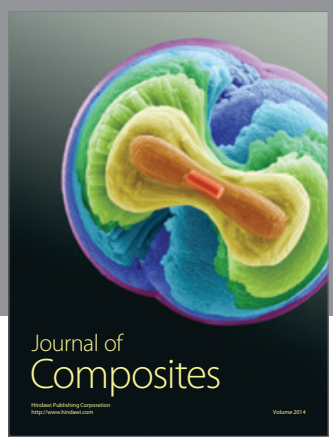
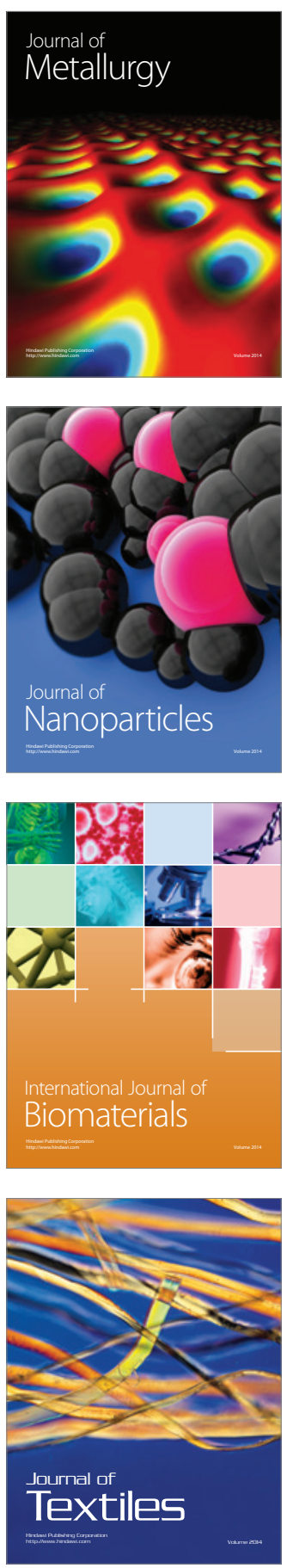